\title{
Imaging Based Detector with Efficient Scintillators for Neutron Diffraction Measurements
}

\author{
Matt W. Seals ${ }^{a}$, Stephen B. Puplampu ${ }^{a^{*}}$, Dayakar Penumadu ${ }^{a}$, Richard A. \\ Riedel $^{b}$, Jeff R. Bunn ${ }^{b}$, Christopher M. Fancher ${ }^{b}$ \\ ${ }^{a}$ Department of Civil and Environmental Engineering, University of Tennessee, Knoxville, TN \\ 37996, USA \\ ${ }^{\mathrm{b}}$ Oak Ridge National Lab, Oak Ridge, TN 37892, USA \\ email: dpenumad@utk.edu
}

(*) corresponding author

\begin{abstract}
The Anger Camera developed by the detector group at the Oak Ridge National Laboratory was utilized for the present work for its unique advantage of employing multiple modules to obtain large active measurement area for detecting diffracted/scattered thermal neutrons. Considering the relatively small flux associated with diffracted/scattered neutrons, suitable efficiency with high spatial resolution is a requirement for utilizing two-dimensional imaging detectors. The potential to implement pulse shape (in addition to pulse height) discrimination-based scintillators further enhances the ability to detect diffracted neutrons with improved signal to noise ratio. In this paper, initial results associated with 6Li glass-based scintillator will be presented. The authors explored the feasibility of using this system to detect and quantify diffraction peaks and peak shifts at the Neutron Residual Stress Facility (NRSF2), High Flux Isotope Reactor (HFIR) in the Oak Ridge National Laboratory (ORNL). Suitable camera mounting and shielding had to be developed. Reference measurements using polycrystalline powders with known atomic planar spacing will be discussed along with measurement settings associated with expected resolution for peak shift measurements. Initial results are promising and demonstrate that a suitable scintillation-based neutron detecting system is viable for residual stress-based diffraction measurements. Small area detectors are also feasible with suitable consideration to scattering volume and distance to detector.
\end{abstract}

\section{User Acknowledgments}

This research [or, A portion of this research] used resources at the High Flux Isotope Reactor [and/or Spallation Neutron Source, as appropriate], a DOE Office of Science User Facility operated by the Oak Ridge National Laboratory.

\section{Introduction}

Anger camera is a variant within the CCD imaging family that allows for fast read-out of large multi-anode photo-multiplier tubes [1-3]. Instead of approaching the issue of readout by establishing a channel for each anode, Anger camera reduces the entire output of the PMT down to four channels allowing for fast data acquisition over the entire photo-sensitive area in realtime. The method by which this is performed is by injecting the output signal of the PMT onto a resistive network when a particle is incident on the scintillator of the PMT as depicted in Figure 1. 


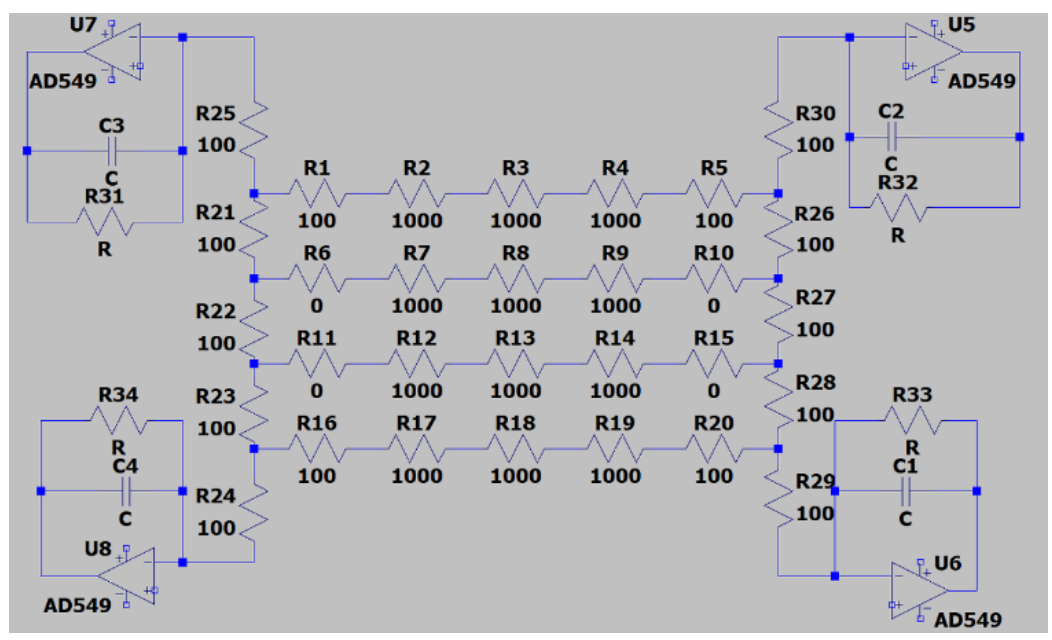

Figure 1: 4x4 Anger logic resistive network

The resistive network is what performs the Anger logic; the position of incidence of a particle on the scintillator can be obtained by the output of the PMT onto the network. Any response from the PMT results in a response at the four outputs of the network in the form of a voltage. These four voltages, $V_{a}, V_{b}, V_{d}$, and $V_{c}$ can be used to determine the location of incidence as a coordinate pair (X, Y) via the formulae Eq. 1 and Eq. 2:

\begin{tabular}{|c|c|}
\hline$X_{\text {position }}=\frac{\left(V_{a}+V_{b}\right)-\left(V_{c}+V_{d}\right)}{V_{a}+V_{b}+V_{d}+V_{c}}$ & (Eq. 1) \\
\hline$Y_{\text {position }}=\frac{\left(V_{a}+V_{d}\right)-\left(V_{c}+V_{b}\right)}{V_{a}+V_{b}+V_{d}+V_{c}}$ & (Eq. 2) \\
\hline
\end{tabular}

The scintillating material used in this study is a $6 \mathrm{Li}$ glass scintillator which has found extensive use in the field of neutron detection [4-6]. Other neutron detection methods lack spatial resolution, and many are by default one dimensional only looking at the number of neutrons that interact with the detector about the vertical channels since the study of neutron diffraction is primarily concerned with the angle at which the bulk of neutrons are diffracting. Typically, the detector type used is the helium-3 tube which is large with a facing size of up to an inch or more in diameter per anode. This results in quite low spatial resolution and a large form-factor.

The Anger camera allows for quite high spatial resolution since it incorporates as many as 1024 anodes into a $116 \mathrm{~mm}$ by116 mm area. In this study, authors seek to implement an Anger camera in diffraction mode for residual stress measurements. Steps towards this implementation require ability to achieve precise, repeatable angular motion and positioning. Furthermore, pulse shape discrimination provides significant assistance in noise reduction however appropriate shielding is still necessary for diffraction. Instrument calibration is also needed to correctly process acquired data. Typically, calibration requires alignment scans in the diffraction plane and acquisition of diffraction peaks from well-known standard powders; additional considerations have to account for the geometry of the detector being a flat surface moving along an arc.

\section{Experimental Method}

To set up and operate the Anger camera at the NRSF2 instrument, additional components had to be put together to ensure accuracy and repeatability of measurements. A stage for the anger camera was secured to the side of the NRSF2 detector; a rotational degree of freedom on the mounted stage, allows the camera to be precisely pointed at the diffracting sample. In addition, the height was carefully adjusted to make sure the detector lied in the horizontal plane of the 
incident beam. Shielding was another significant component; its purpose is to prevent background radiation from striking the detector and ensuring the Anger camera only "sees" the sample on the NRSF2 stage. Shielding schematics are shown in Figure 2:

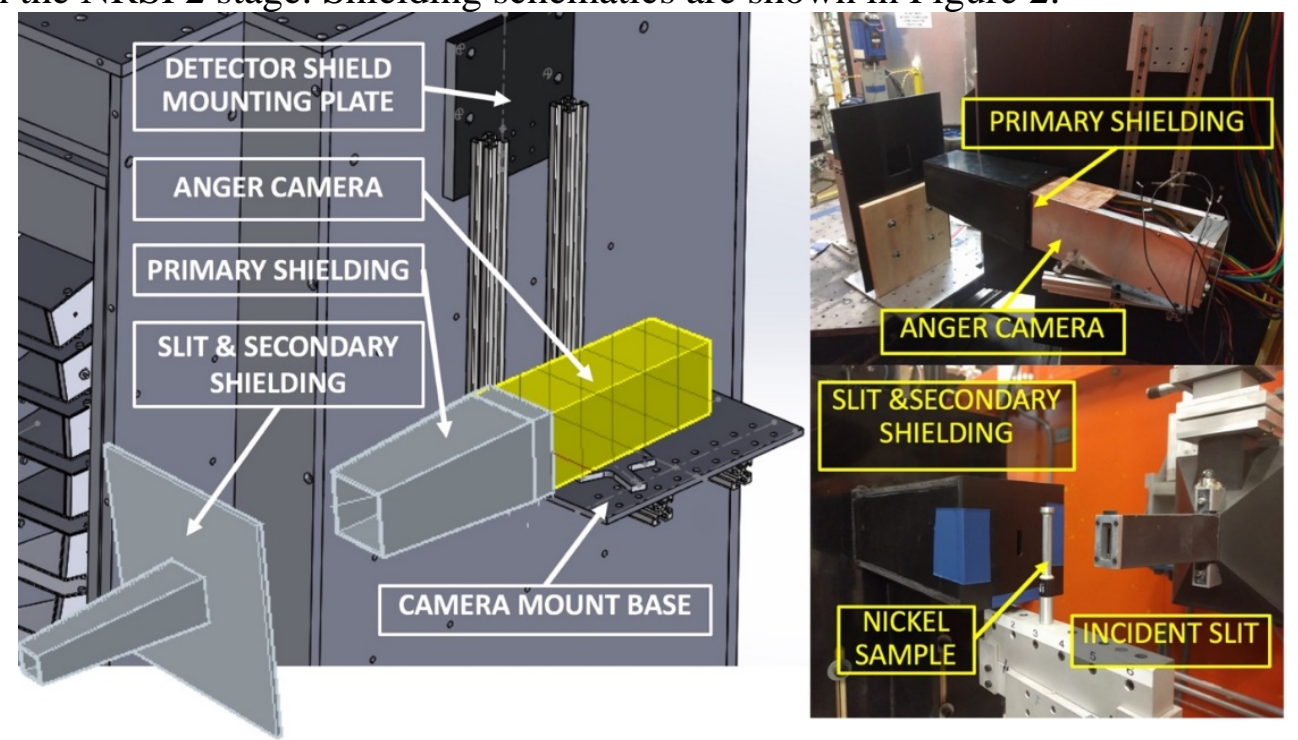

Figure 2: Schematic representation and actual pictures of shielding components and camera mounted on NRSF2 detector

Due to special constrains and the operating distance of the camera from the diffracting sample, shielding could not be implemented as a single snout going from the detector to the sample; as a solution, shielding was split into two parts such that the overall effect reproduces the effect of a single snout. The Anger camera was connected to and integrated with the NRSF2 control workstation. This enabled us to control both instruments from one computer. Reference data is obtained from the main instrument then, having a set angular offset between the Anger camera and the NRSF2 detector, the main detector is driven to a position such that the Anger camera can record the same peak.

\section{Results}

Imaging data collected using Anger camera comes in the form of raw binary packets consisting of 262144 or 65536 16-bit integer values, depending on the number of PMT's utilized. Using a full 4-array of PMT’s results in a 512x512 image as shown in Figure 3(a).
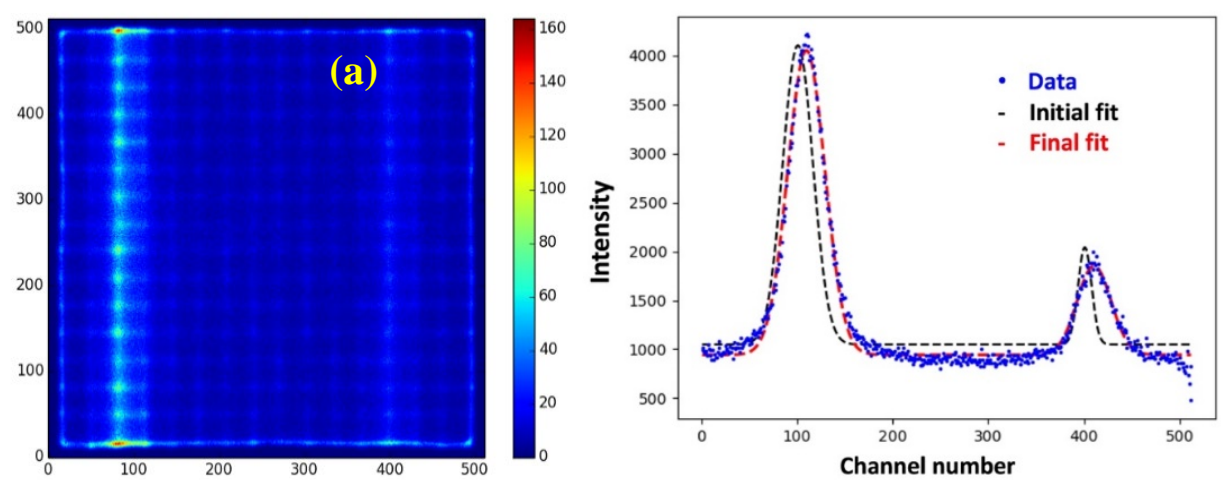

Figure 3: (a) example heat-map image from Anger camera depicting two peaks; colormap shows counts per pixel over image acquisition time (b) Fitted vertical integration

The processing of the data involves converting the raw pixel array into a 16-bit image using the Python Imaging Library. The data is then integrated vertically such that if the image pixels 
are expressed in row-column format as $I(M, N)$ then the value for each channel according to Eq. 3 will be:

$$
Y_{N}=\sum_{i=0}^{N} I(m, i) \text { where } m \in M
$$

(Eq. 3)

Where $Y_{N}$ indicates the number of neutrons counted on a channel as a result of the vertical sum of the pixels on that channel. Figure 3(b) was processed to obtain the plot shown in Figure 3(b) where a Gaussian peak fit is implemented. Peak information such as Signal to Noise Ratio (SNR) and full-width-at-half-max (FWHM) can be used to describe the performance of the camera in combination with different scintillators while looking at neutron scattering from various reference samples. All acquired images were normalized to remove noise due to background and detector intensity variations. Dark current (recording data with the shutter closed) and open beam images (recording data with open shutter and vanadium incoherent scatterer in place of the sample) were recorded and used for the normalization process. Images were normalized according to Eq. 4 where $I_{R A W}$ is the intensity of the recorded image, $I_{D C}$ is the intensity of the dark field image and $\mathrm{I}_{\mathrm{OPEN}}$ is the intensity of the open beam image.

$$
I_{R A W}=\frac{I_{R A W}-I_{D C}}{I_{O P E N}-I_{D C}}
$$

Initial measurements consisted of standard powder samples diffraction peaks. These samples are used the NRSF2 instrument calibration at the beginning of each cycle. Single peaks were acquired for Body Centered Cubic Iron, Germanium, Nickel and Inconel samples. Count time was 60 seconds for each measurement. Example normalized images are shown in Figure 4 with superimposed intensity vs. channel plot obtained from vertical image integration.

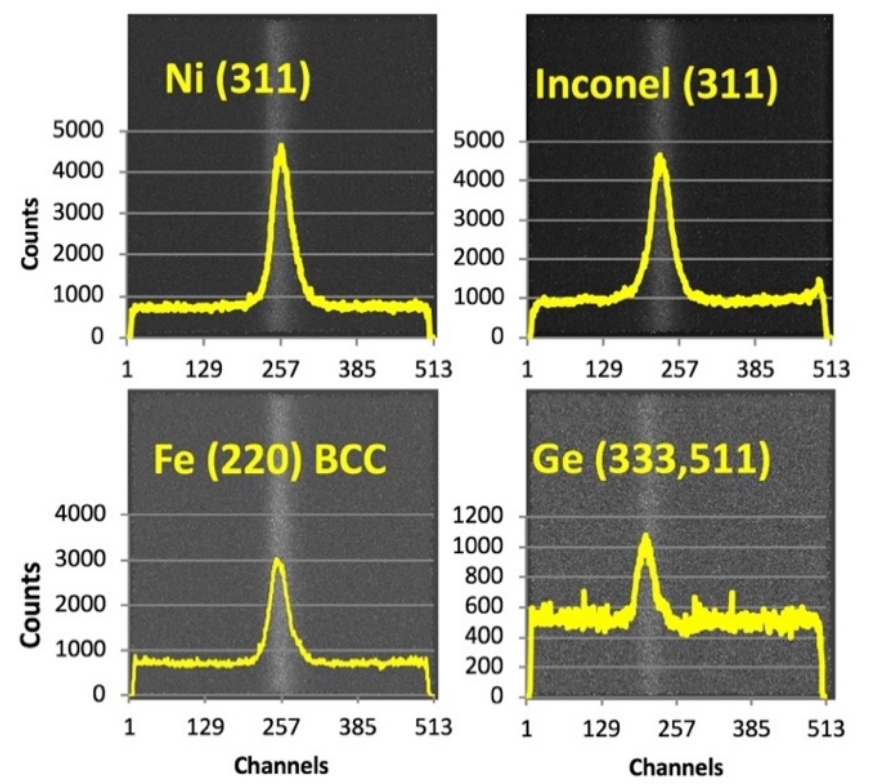

Figure 4: Normalized two-dimensional imaging of diffraction peaks with superimposed intensity plot 


\section{Discussion}

Diffraction peaks were selected for $2 \theta$ values as close to $90^{\circ}$ as possible; for quantification of these measurements, curvature was neglected and, subsequent to normalization, raw intensity was obtained by vertical image integration. To determine detector resolution, a $2 \theta$ sweep using an Inconel powder sample was performed. During this set of scans, two peaks swept across the detector. All obtained images were integrated and peak positions within the field of view of the camera were determined by the channel number. The channel number peak positions are then plotted versus angular $2 \theta$ position as shown in Figure 5 and the slope yields the channel per degree value giving an idea of what the spatial resolution of the detector is for the given operating sample to detector distance. For the operating distance of $750 \mathrm{~mm}$, the Anger camera was found to have an approximate resolution of 57 pixels per degree.

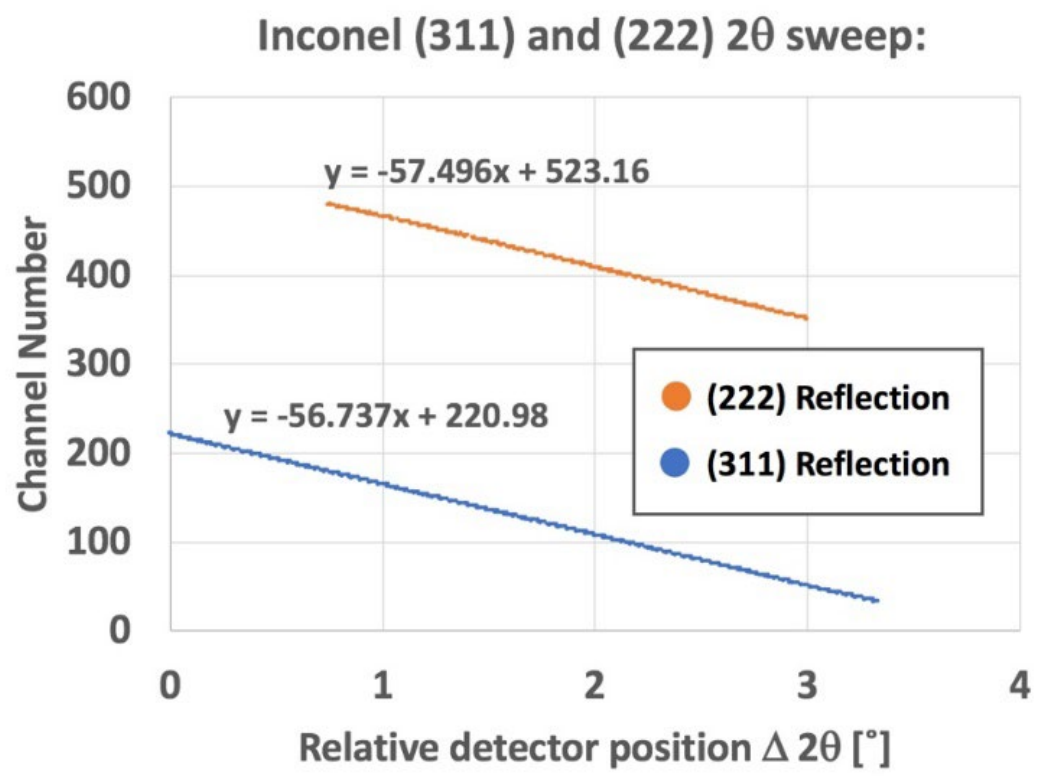

Figure 5: Peak position (channel) vs. $2 \theta$ position

\section{Conclusions}

Integration of the Anger camera in a diffraction instrument was successful. Development and implementation of shielding was part of the study and performed as intended (system performance without shielding was not investigated). For portable applications, a smaller footprint would be desirable however shielding dimensions are geometrically determined by sample to detector distance. Overall the Anger camera proved to be a viable instrument for neutron diffraction measurements. The results show a high degree of 2-D spatial resolution and for the working distance of $750 \mathrm{~mm}$ the Anger camera was found to cover a $2 \theta$ range of approximately $8^{\circ}$. Future work will be aimed at evaluating the possibility of using the Anger camera for residual strain measurements; it will be critical to determine the magnitude of the strain change that can be determined by processing the two-dimensional images recorded by the Anger camera and successfully resolving shifts in peak position.

\section{References}

[1] R. A. Riedel, C. Donahue, T. Visscher, and C. Montcalm, "Design and performance of a large area neutron sensitive anger camera," Nuclear Instruments and Methods in Physics Research Section A: Accelerators, Spectrometers, Detectors and Associated Equipment, vol. 794, pp. 224-233, 2015. https://doi.org/10.1016/j.nima.2015.05.026 
[2] P. D. Olcott, J. A. Talcott, C. S. Levin, F. Habte, and A. M. K. Foudray, "Compact readout electronics for position sensitive photomultiplier tubes," IEEE Transactions on Nuclear Science, vol. 52, no. 1, pp. 21-27, 2005. https://doi.org/10.1109/TNS.2004.843134

[3] S. Siegel, R. W. Silverman, S. Yiping, and S. R. Cherry, "Simple charge division readouts for imaging scintillator arrays using a multi-channel PMT," IEEE Transactions on Nuclear Science, vol. 43, no. 3, pp. 1634-1641, 1996. https://doi.org/10.1109/23.507162

[4] L. M. Bollinger, G. E. Thomas, and R. G. Ginther, "Glass Scintillators for Neutron Detection," Review of Scientific Instruments, vol. 30, no. 12, pp. 1135-1136, 1959. https://doi.org/10.1063/1.1716471

[5] J. M. Neill, D. Huffman, C. A. Preskitt, and J. C. Young, "Calibration and Use of a 5-Inch Diameter Lithium Glass Detector," (in English), Nuclear Instruments \& Methods, vol. 82, pp. 162-\&, 1970. https://doi.org/10.1016/0029-554X(70)90343-5

[6] C. Coceva, "Pulse-shape discrimination with a glass scintillator," Nuclear Instruments and Methods, vol. 21, pp. 93-96, 1963. https://doi.org/10.1016/0029-554X(63)90092-2 